\title{
Interpersonal violence and mental health outcomes following disaster
}

Robyn Molyneaux, Lisa Gibbs, Richard A. Bryant, Cathy Humphreys, Kelsey Hegarty, Connie Kellett, H. Colin Gallagher, Karen Block, Louise Harms, John F. Richardson, Nathan Alkemade and David Forbes

\section{Background}

Disasters pose a documented risk to mental health, with a range of peri- and post-disaster factors (both pre-existing and disasterprecipitated) linked to adverse outcomes. Among these, increasing empirical attention is being paid to the relation between disasters and violence.

\section{Aims \\ This study examined self-reported experiences of assault or violence victimisation among communities affected by high, medium, and low disaster severity following the 2009 bushfires in Victoria, Australia. The association between violence, mental health outcomes and alcohol misuse was also investigated.}

\section{Method}

Participants were 1016 adults from high-, medium- and lowaffected communities, 3-4 years after an Australian bushfire disaster. Rates of reported violence were compared by areas of bushfire-affectedness. Logistic regression models were applied separately to men and women to assess the experience of violence in predicting general and fire-related post-traumatic stress disorder, depression and alcohol misuse.

\section{Results}

Reports of experiencing violence were significantly higher among high bushfire-affected compared with low bushfireaffected regions. Analyses indicated the significant relationship between disaster-affectedness and violence was observed for women only, with rates of 1.0, 0 and $7.4 \%$ in low, medium and high bushfire-affected areas, respectively. Among women living in high bushfire-affected areas, negative change to income was associated with an increased likelihood of experiencing violence (odds ratio, 4.68). For women, post-disaster violence was associated with more severe post-traumatic stress disorder and depression symptoms.

\section{Conclusions}

Women residing within high bushfire-affected communities experienced the highest levels of violence. These post-disaster experiences of violence are associated with post-disaster changes to income and with post-traumatic stress disorder and depression symptoms among women. These findings have critical implications for the assessment of, and interventions for, women experiencing or at risk of violence post-disaster.

\section{Declaration of interest}

None.

\section{Keywords}

Post-traumatic stress disorder; trauma; violence.

\section{Copyright and usage}

(c) The Royal College of Psychiatrists 2019. This is an Open Access article, distributed under the terms of the Creative Commons Attribution-NonCommercial-ShareAlike licence (http://creativecommons.org/licenses/by-nc-sa/4.0/), which permits non-commercial re-use, distribution, and reproduction in any medium, provided the same creative Commons licence is included and the original work is properly cited. The written permission of Cambridge University Press must be obtained for commercial re-use.
Whether primarily natural or human-made, disasters pose a welldocumented risk to mental health, with increased rates of depression, post-traumatic stress disorder (PTSD) and related conditions among those in affected areas. ${ }^{1}$ Research has also thoroughly examined the pre-, peri- and post-disaster risk factors that contribute to the prevalence of mental health issues, ${ }^{1,2}$ with a range of life stressors (both pre-existing and disaster-precipitated) linked to adverse outcomes. ${ }^{1,3,4}$ One particular focus in existing literature is interpersonal violence following disasters (a broad term to cover all types of violence committed by one person against another). ${ }^{5}$ This is often classified at the subcategorical level, such as gender-based violence (violence against someone based on socially ascribed gender roles), community violence (violence between unrelated individuals, who may be known or unknown to each other) or family violence (violence largely between family members and intimate partners), an aspect of which is intimate partner violence (IPV; violence directed against an intimate partner or ex-partner). ${ }^{5,6}$ There is growing empirical evidence of gender-specific patterns of vulnerability to violence following natural disasters. ${ }^{7-9}$ Longitudinal research indicates that experiences of IPV before a disaster increase the likelihood of IPV following a disaster event, ${ }^{10}$ and this may be exacerbated by heightened stress post-disaster and damage to the physical and social infrastructures that provide domestic violence response and support. ${ }^{11}$ Although previous research has found support both for $^{11,12}$ and against ${ }^{10,13}$ post-disaster increases in IPV, certain peridisaster experiences (such as property damage, ${ }^{8,14}$ job loss ${ }^{15}$ and financial hardship ${ }^{16}$ ) have been linked to increased IPV risk. This is a significant mental health issue, as post-disaster IPV is associated with $\mathrm{PTSD}^{8,14}$ and depression. ${ }^{12,17}$ One gap within previous literature is that there has been little consideration of the pattern of interpersonal violence across communities variably affected by disaster. In the absence of pre-disaster data on incidence of violence, such comparative data on impact severity could indicate the extent of influence of the disaster experience, and identify those individuals and communities that may be at increased risk of certain post-disaster experiences and poorer mental health.

In February 2009, widespread bushfires in Victoria, Australia resulted in 173 fatalities and the damage or destruction of 3500 buildings. ${ }^{18}$ Known as the 'Black Saturday' bushfires, they remain one of Australia's worst disasters on record and produced longterm repercussions for the functioning and well-being of affected communities. ${ }^{3}$ This paper aimed to fill a gap in the current research by identifying those at the most risk of interpersonal violence in the post-disaster setting, factors that relate to experiences of postdisaster violence and how these experiences of violence may affect individuals' health and well-being. First, this paper compared rates of violence across communities that experienced low, medium and high bushfire-affectedness following these widespread bushfires (for 
the total sample, and by gender). ${ }^{18}$ Second, the paper examined the relationship between the occurrence of violence and other negative experiences that occurred following the fires among those individuals reporting the highest levels of violence. ${ }^{8,14-16}$ Finally, this paper investigated the gender-specific relationship between interpersonal violence and well-being and mental health (linked to violence and/or disaster exposure ${ }^{8,12,14,17,19-22}$, with particular respect to post-disaster depression, PTSD and heavy drinking.

\section{Method}

\section{Participants}

The sample comprised 1016 residents (aged $\geq 18$ years) from 25 communities across 10 Victorian rural and regional locations. The data were gathered in 2012-2013 as part of the Beyond Bushfires: Community Resilience and Recovery study, in which 25 communities in 10 locations were selected to represent diversity in terms of bushfire-affectedness, socioeconomic status, community size and remoteness from the central business district of Melbourne, Victoria $^{23}$ (www.beyondbushfires.org.au). Census data from 2006 indicated a total adult population of 7693 in the selected communities. The contact details of current residents in the selected communities and those relocated since the fires $(N=7467$ adults $)$ were obtained from the Victorian Electoral Commission, which approved one personalised letter of invitation to the study (including a postage-paid return envelope) that was sent to this sample. Community awareness activities, mailbox drops, region-based phone calls, news and social media activities were conducted to increase awareness of the study and methods for registration. All participants provided informed consent. Of those individuals who were eligible to take part in this study, $14.1 \%$ eventually participated and completed the survey $(N=1056)$, and only those participants who were residents in the selected communities at the time of the bushfires are included in analyses $(N=1016)$.

\section{Measures}

\section{Community-level impact}

Communities were categorised according to community-level exposure. For the purpose of this analysis, the community groupings were adjusted to provide clear cut-offs based on numbers of fatalities, resulting in three distinct groups. These groups were defined as high bushfire-affectedness (operationalised as four or more fatalities, and high numbers of houses lost; $n=675$ ), medium bushfire-affectedness (significant amount of property damage, with the most severe end of the medium grouping including one or two fatalities; $n=136$ ) and low bushfire-affectedness (no evidence of direct burning; however, communities may have been on fire alert during the bushfires and affected by road closures and involvement of local firefighters in the regional response; $n=$ 205). This categorisation system utilised housing damage figures provided by the Victorian Government Rapid Impact Assessment process and fatalities by community as advised by the Victorian Coroner. Our approach is consistent with the use of fatalities and residential housing loss as community-level impact indicators by the Victorian Bushfire Reconstruction and Recovery Authority.

\section{Bushfire exposure}

Three items were used to assess individual-level bushfire exposure: whether a respondent feared for their life (yes/no), whether the respondent experienced loss of a loved one (yes/no) and extent of property loss owing to the fires (on a scale from 0 (nothing) to 10 (everything)).

\section{Experience of assault or violence}

Participants were asked whether, since February 2009 (the 2009 bushfires), they had personally experienced assault or violence (yes/no).

\section{PTSD}

Probable PTSD was assessed with the Posttraumatic Stress Disorder Checklist-Civilian Version (PCL). ${ }^{24}$ This four-item version of the PCL comprises four items each scored on a five-point Likert scale that index key symptoms of PTSD over the previous 4 weeks (Cronbach's alpha $=0.82$ ). The PCL was altered to ask whether the symptom being endorsed was in response to reminders of the Black Saturday fires (PCL Fire-related), or in response to reminders of traumatic events generally (PCL General). Total scores for each participant were then generated for both Fire-related and General post-traumatic stress symptoms.

\section{Depression}

Probable major depression was assessed using the Patient Health Questionnaire (PHQ- ${ }^{25}$ ). Presence of depression was coded if five of the nine symptoms had been present for most days in the previous 2 weeks (Cronbach's alpha $=0.86$ ).

\section{Alcohol use}

Alcohol use was assessed with the three-item self-report Consumption Scale of the Alcohol Use Disorder Identification Test (AUDIT-C, an abbreviated version of the AUDIT ${ }^{26}$ ), assessing how often the respondent drinks, how many drinks are consumed in a day and how often they have six or more drinks in a single occasion, which measures the amount of alcohol consumption but not adverse effects of alcohol use (Cronbach's alpha $=$ $0.81)$. Items are summed to produce a score of $0-12$ ( 0 reflects no alcohol use).

\section{Major life stressors}

Participants were asked to indicate whether they had experienced major life stressors in the form of disruptions to their income, employment status, occupation, accommodation or personal relationships since the bushfires, and whether these had resulted in a negative outcome.

\section{Procedure}

The study was approved by the University of Melbourne Human Research Ethics Committee (HESC ID: 1034829.4). Data collection was piloted in late 2011, then conducted between April 2012 and January 2013. The full study protocol, including a participatory approach and strategies to ensure post-trauma sensitivity, are described elsewhere. ${ }^{23,27}$ Surveys were either self-completed via an online questionnaire or administered via computer-assisted telephone interview (CATI), depending on participant preference. The survey commenced with a range of sociodemographic questions, followed by the PHQ-9, AUDIT-C, PCL and questions about bushfire exposure and subsequent traumatic or stressful life events.

The Beyond Bushfires: Community, Resilience and Recovery study initially sought to recruit approximately equal numbers from low, medium and high bushfire-affected communities. However, population estimates for these communities indicated a larger population across the high bushfire-affected communities (compared with the medium and low bushfire-affected communities), and this was further enhanced by the higher response rates within those communities. As such, the final sample had an overrepresentation of individuals from high bushfire-affected communities. Based on the total adult population for each of these regions, the 
participation rates were approximately 9.5\% for low, 9\% for medium and $19.2 \%$ for high bushfire-affected communities (based on population estimates of 2153, 1532 and 3508, respectively ${ }^{23}$ ). Communities were selected with a view to equivalence across regions in Socio-Economic Indexes for Areas, community size and remoteness (i.e. distance from Melbourne, Victoria's state capital and most populous city). When compared with census data from the participating communities, ${ }^{28}$ the study sample was found to have a significant overrepresentation of women $\left(\chi^{2}(1)=\right.$ 51.36, $P<0.001)$ and was older than the population data suggests for residents in these regions $\left(\chi^{2}(7)=741.86, P<0.001\right)$. This pattern of differences was also observed for each region separately, with samples in the low, medium and high bushfire-affected communities demonstrating an overrepresentation of women and older residents compared with population estimates. Two key mental health measures (the PHQ-9 and PCL-4, detailed below) were assessed for measurement invariance between the two interview modes (CATI and online), controlling for age and gender, and found to be completely scalar invariant, with no mean difference between the two groups. This indicates that the two modes of survey completion produced consistent results.

\section{Statistical analyses}

Analyses were conducted with SPSS version 24 for Windows. The first stage of analysis compared gendered rates of violence within communities that experienced low, medium and high bushfireaffectedness. $\chi^{2}$ analyses were used to investigate the relationship between region bushfire-affectedness, gender and experiences of violence.

The second stage of analysis examined the relationship between the occurrence of violence and other negative experiences that occurred following the fires among those individuals at high risk of experiencing violence, based on the analysis of gendered rates of violence across communities. A binomial logistic regression was conducted to examine the risk of major life stressors (negative change in income, employment, occupation, accommodation and relationship) in the prediction of experiences of violence among the high-risk group/s identified in the preceding analysis. In recognition of the smaller sample size, a parsimonious model was created by utilising $\chi^{2}$ analyses to identify statistically significant relations between violence and major life stressors (negative change in income, employment, occupation, accommodation and relationship). Significant variables were then entered into the regression model.

Finally, this paper investigated the gender-specific influence of experiences of violence on individuals' well-being, with particular respect to post-disaster depression, PTSD and heavy drinking for participants across all communities. Separate multiple regressions with a function to account for the effects of clustering to correct standard errors were conducted in Mplus version 7 for Windows (Muthén \& Muthén, Los Angeles, CA; see https:// www.statmodel.com/). ${ }^{29}$ This approach generates standard error estimates that more accurately reflect the variability in the regression coefficient estimates when there is clustering in the data. ${ }^{30}$ These regressions were conducted with participants from all communities to predict symptom severity for depression and PTSD, and heavy drinking. These analyses controlled for gender, age at the time of the Black Saturday fires, highest level of education and (individual) bushfire exposure. Experiences of violence were then entered to index the additional effects on psychological outcomes and drinking behaviours. Models were run separately for men and women to assess for gender-specific associations, based on the literature.

\section{Results}

The analysis included 967 participants $^{\mathrm{a}}$ with a mean age of 56.5 (s.d. $=13.30$, range, 18.3-87.7). The majority of participants were female $(n=585,60.5 \%)$. Participants were not found to differ between the three levels of affectedness in terms of gender, country of birth or employment status. However, participants in low bushfire-affected regions were on average slightly older than those of high bushfire-affected regions (although the effect size was small; Hedges' $g=0.21, F(244.72)=0.07, P=0.02)$, and fewer participants in the low bushfire-affected communities had tertiary education than those in the medium and high bushfire-affected communities (24.4\% in the low bushfire-affected group, $46.9 \%$ in the medium bushfire-affected group and $35.6 \%$ in the high bushfire-affected group; $\left.\chi^{2}(2, N=967)=16.10, P<0.001\right)$.

Since the 2009 bushfires, $5.3 \%$ of participants in the study reported experiencing assault or violence. Rates of participant reports of violence, and other life stressors experienced since the bushfires separated by region are provided in Table 1. A significant relationship was observed between region and experience of violence $\left(\chi^{2}(2, N=962)=10.68, P=0.005\right)$. An inspection of the adjusted standardised residuals found an overrepresentation of individuals who experienced violence in the high bushfire-affected regions.

Although no significant differences were found in the reports of violence between males and females within each region or across the complete sample, differences emerged across regions. When rates of violence across regions were examined separately by gender, no significant differences in the proportions of reported violence across the three regions was found for males. However, among female participants there was a significant difference across regions $\left(\chi^{2}(2, N=\right.$ $581)=11.67, P=0.003)$, with inspection of the adjusted standardised residuals indicating an overrepresentation of women who experienced violence in the high bushfire-affected region. As seen in Table 1, rates of women experiencing violence since the time of the fires were 1.0, 0 and $7.4 \%$ in low, medium and high bushfireaffected areas, respectively.

\section{Predictors of violence against women in high bushfire-affected regions}

Analyses then explored a series of predictors of experiencing violence among women residing in the high bushfire-affected areas $(n=406$; model results are presented in Table 2$) \cdot \chi^{2}$ analyses were used to assess significant relations between violence and major life stressors. Proposed control variables (education level and relationship status) were not significantly related to experiences of violence, nor were changes in occupation (with a negative outcome), as such these variables were not included in the logistic regression model.

Change in employment (with a negative outcome; $\chi^{2}(1, N=$ $403)=7.15, P=0.007$ ), change in accommodation (with a negative outcome; $\left.\chi^{2}(1, N=403)=9.06, P=0.003\right)$ and change in income (with a negative outcome; $\chi^{2}(1, N=402)=15.97, P<0.001$ ) were

a. As this is a large-scale study, data collection was spread over a number of months. A trend was observed toward participants from low bushfire-affected communities completing the survey earlier. Further analysis (weighted for the different group sizes) indicated that this effect size was small. Upon investigation, the two pilot communities who completed the survey at an earlier time point were producing this trend, and when removed from the sample no differences were observed between timing of survey completion and community impact. As a result, this subset of participants $(n=49)$ are excluded from the initial analysis. Importantly, this produces no change in the significance of findings. Additionally, no relationship was found between the number of days between bushfires and survey completion, and reports of violence. 


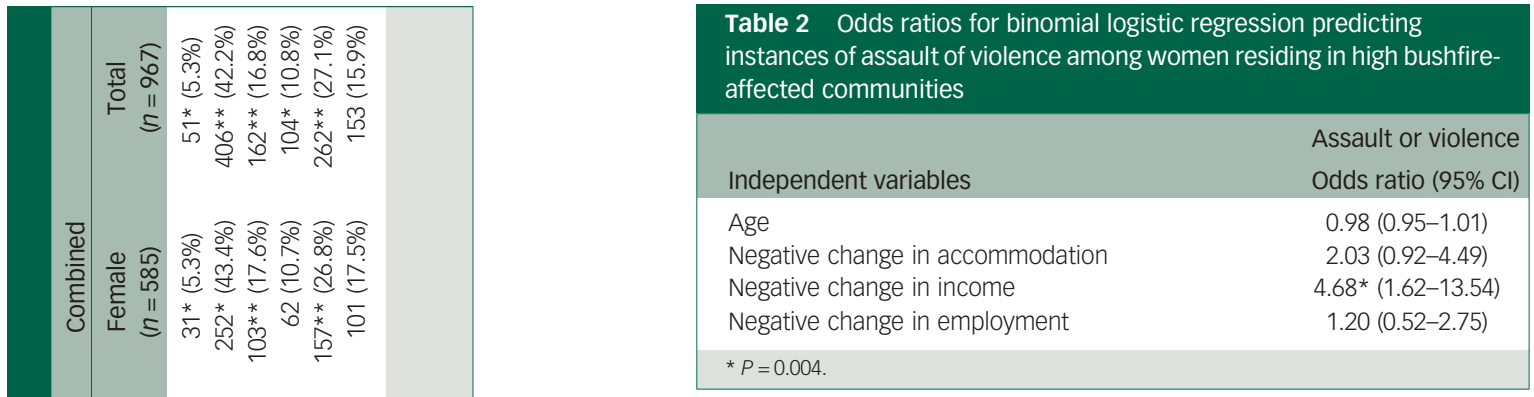

all found to be significantly related to reports of violence, and have endorsement rates within the sample of 31.5, 23.9 and $48.1 \%$, respectively. Participants' age was also found to be significantly related to experiences of violence and was therefore included as a controlling variable. Change in relationship (with a negative outcome; $\left.\chi^{2}(1, N=398)=9.30, P=0.002\right)$ was found to be significantly related to experiences of violence among women in high bushfire-affected communities, showing an overrepresentation of women reporting both a negative change in relationship and an experience of violence. However, the expected and actual count in some cells was very low, as was overall endorsement (12.7\%), and as a result the decision was made to exclude this variable from the logistic regression.

The model significantly predicted reports of violence $\left(\chi^{2}(4)=\right.$ 23.09, $P<0.001)$, and found that experiencing a negative change in income since the fires predicted 4.68 times higher odds of violence among women residing in high bushfire-affected regions (Table 2). Other life stressors were not found to be associated with experiences of violence among this subgroup.

\section{Mental health and heavy drinking across all regions}

Regression weights for the variables in the fire-related and general PTSD, depression and heavy drinking models are presented in Table 3. All variables were assessed for excessive multicollinearity and Variance Inflation Factor (VIF) score ranges were 1.02-1.15 (for both male and female models), which is low and permits simultaneous inclusion in a regression equation. All models were statistically significant.

When accounting for clustering at the community level on standard errors, experiences of violence were found to predict increased depression symptoms and more severe PTSD symptoms among women but not men. As anticipated, fire exposure variables (loss of a loved one, property loss and fear for life) each uniquely contributed to increased fire-related PTSD symptoms among both men and women. Interestingly, after accounting for the predictive value of these variables, experiencing violence since the fires significantly predicted increased fire-related PTSD among women, but not among men. When community clustering was accounted for, experiences of violence were not found to predict drinking behaviours among men or women.

\section{Discussion}

This study provides the first evidence of the overrepresentation of individuals reporting post-disaster experiences of violence in high disaster-affected communities compared with communities with lower levels of disaster-affectedness. Closer examination of this association reveals that the relationship between severity of impact and experiences of violence appears to be confined to women, with $7.4 \%$ of women in high bushfire-affected areas reporting experiencing violence in the 3-4 years following the bushfires, 


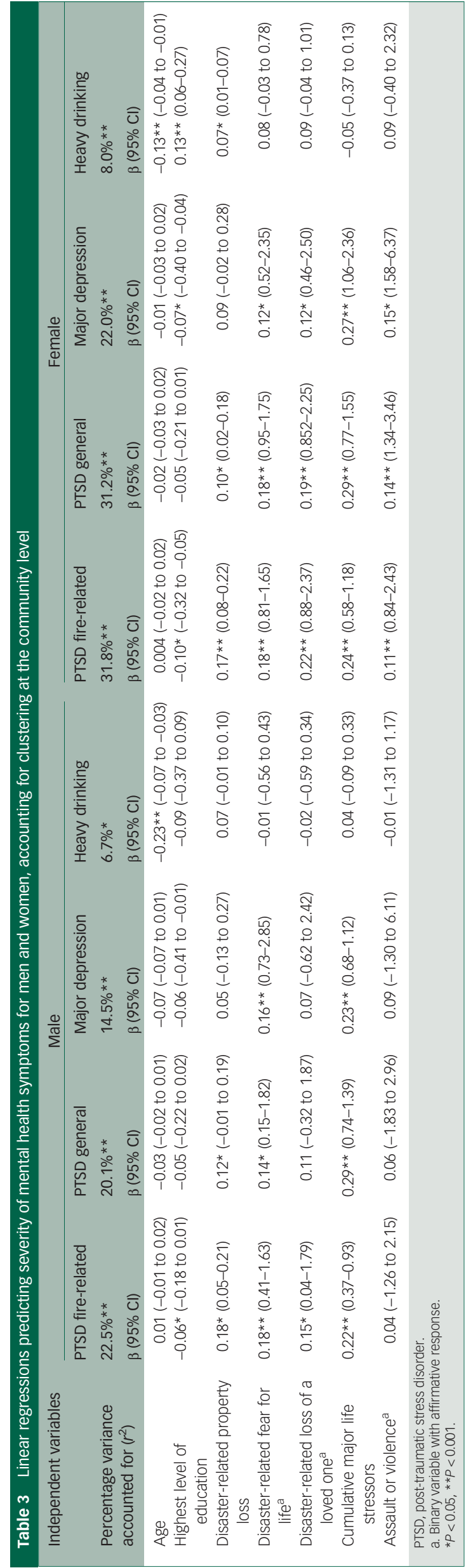

compared with 0 and $1.0 \%$ in medium and low bushfire-affected areas, respectively. These findings of higher rates of reported violence against women in regions highly affected by bushfires extends existing literature on the increased prevalence of general interpersonal violence and IPV following disasters. ${ }^{8}$ Subsequent analyses of potential factors associated with increased risk of violence in women in high bushfire-affected areas (i.e. age, education and stressors including negative changes in income, employment, occupation, accommodation or relationship) showed that only negative income change following the bushfires was significant, accounting for a fourfold increase in risk. It appears, therefore, from this study that reduced income adds increased risk for women's experience of violence in the post-disaster context.

Consistent with existing literature, a relationship was observed between experiences of violence and poor mental health. Experiences of violence since the bushfires were associated with increased symptoms of PTSD and depression among women 3-4 years after the disaster, even when controlling for the predictive validity of potentially traumatic fire-related experiences and other life stressors. These results reflect those reported elsewhere for women in the post-disaster context (both men and women, ${ }^{2,8,21}$ and women specifically ${ }^{12,14,31,32}$ ) and in literature on the relationship between post-disaster life stressors and mental health. ${ }^{3,4,33}$ Among men, experiences of violence were not found to be associated with PTSD or depression; this did not reflect existing research on the observed association between experiences of violence and PTSD (for both men and women, ${ }^{2,8}$ and men ${ }^{34}$ specifically) and depression. ${ }^{21}$ It should also be considered that given the cross-sectional nature of this data, those experiencing poor mental health may be at increased risk of experiencing violence. Further longitudinal research would be required to clarify the direction of this relationship.

The unique profiles of association between experiences of violence and mental health outcomes for men and women could be the product of gender-specific experiences of violence. As reflected in the existing literature, gender-based forms of violence (such as sexual violence and IPV) increase in the acute and longer-term aftermath of a natural disaster. ${ }^{8,12,35}$ A range of factors may contribute to this, such as pre-disaster experiences of violence, ${ }^{10}$ specific peri-disaster and post-disaster experiences and stressors, ${ }^{8,14-16}$ relationship adjustment ${ }^{31}$ and non-physical forms of intimate partner abuse (verbal and psychological ${ }^{8}$ ). The findings from this study identify reductions in income as the most salient stressor with respect to this risk.

The findings of this study have implications for the assessment of risk and prevention of disaster related violence, especially among women. In particular, this research indicates that women should be considered for preventative and post-disaster support and interventions regarding violence within communities at risk of, or recently experiencing, bushfires. That the strongest predictor of women's experience of violence in the high bushfire-affected area was a negative change in income suggests that subsequent financial stress ought to be identified as a risk factor in post-disaster violence. However, it should be acknowledged that because of the cross-sectional nature of this data, it is not possible to identify the exact causal relationship between income and experiences of assault; it is also plausible that both violence and mental health may be influenced by underlying socioeconomic deprivation in the post-disaster setting and that experiences of violence may cause women to leave relationships, in turn negatively affecting the women's income. In addition, in the context of data from previous research that links pre-disaster and post-disaster victimisation, ${ }^{10}$ these findings highlight the need raise awareness and to monitor and support those members of the community already at risk of IPV. This is especially important considering the gender-specific burden of violence on 
mental health, which has pressing implications for women's ability to recover from disaster experiences in the longer term.

In conclusion, this study examined prevalence of violence across regions with varying levels of disaster impact. It highlighted important gender distinctions in both the experience of violence, and the association between violence and mental health post-disaster. The findings showed an increased post-disaster risk of violence for women residing in high bushfire-affected regions compared with lesser affected areas. Negative changes to income (a core feature of the social disruption that follows a major disaster) was identified as a particularly important indicator of risk and may provide a target for intervention. Furthermore, reports of violence among women increased the severity of mental health symptoms, suggesting that post-disaster experiences of violence may impair women's ability to recover from disasters. These findings have critical implications for future research and for the assessment of, and interventions for, women experiencing or at risk of violence in the post-disaster context.

\section{Limitations}

The authors acknowledge several limitations to this study. First, to permit comparison between variably affected communities, a system of categorisation of community exposure was formulated at the community level, based on severity of bushfire damage to property and fatalities. This measure did not incorporate individual exposure variables (although these were measured separately, and included in analysis of the third research question). With regards to response rates within these community groupings, it is acknowledged that response rates were lower for low bushfire-affected communities (approximately 9.5\% for low bushfire-affected, 11.8\% for medium bushfire-affected and $18 \%$ for high bushfire-affected communities), and this results in an overrepresentation of individuals from more highly bushfire-affected communities within the study sample. Although all efforts were made to ensure equivalence across regions in Socio-Economic Indexes for Areas, community size and remoteness when recruiting from communities, it is possible that these response rates may bias the sample. When compared with census data from the participating communities (Australian Bureau of Statistics, 2011 community profiles), the study sample as a whole was found to have a significant overrepresentation of women and was older than the population data suggests for residents in these regions. This pattern was also reflected across the low, medium, and high bushfire-affected communities separately. As limited data were collected relating to individual sociodemographic factors, it was not possible to compare these in detail across community impact groups within the data-set, and as such it must be acknowledged that the results of this study may reflect a more general relationship between violence and socioeconomic deprivation. Additionally, although overall sample size for this study was large, because of the overrepresentation of women and participants from high bushfire-affected communities and rates of violence reported, some groups within the analysis were small. This limitation restricted the analysis and number of independent variables that could be investigated, and so further research should investigate these relationships within a larger sample.

Second, the measure used in this study to assess violence was general in nature and did not ask participants to provide any detail on the type of violence they experienced, its repetition or frequency, who perpetrated it or whether experiences of violence were present before the bushfires. As such, it is not possible to conclude whether the violence they experienced was perpetrated by someone known to them (such as a partner, constituting IPV), the gendered nature of this relationship or someone unknown to the respondent. Depending upon the individual respondent's interpretation of the question and their personal experience, it is possible that this broad measure of violence may have led to underreporting of violence (or less probable, but possible overreporting). Future research should seek to investigate this relationship among specific forms of violence more closely, as it may shed light on the gender-specific vulnerabilities to violence in the post-disaster setting (in line with other literature ${ }^{7}$ ). In line with this, research should also consider the role of family violence and violence against children. Additionally, it should be acknowledged that PTSD was assessed using a short form four-item version of the PCL, although this has been shown to have good diagnostic efficiency in the literature. ${ }^{24}$ Third, as previously noted, the data used in this study were cross-sectional, therefore causality cannot be inferred between reported experiences of post-disaster violence and the development of the assessed mental health conditions and heavy drinking. This also applies to the relationship between negative changes to income and experiences of violence. Finally, it is acknowledged that the data used within this paper represents individuals who are clustered within communities. Although there are many ways in which to analysis such data, one of which was used in this paper, ${ }^{30}$ future research would be well placed in examining such relationships between community-level disaster impact and experiences of violence through more involved hierarchical or multilevel forms of modelling, to best represent this inherent structure. Although acknowledged as a limitation of this current study, it is believed that the findings presented here provide important insight into violence post-disaster that has not been reported previously. It is hoped that further research will further investigate the incidence and nature of violence in the post-disaster setting.

Robyn Molyneaux (D), Research Fellow, Child and Community Wellbeing Unit, Centre for Health Equity, University of Melbourne, Australia; Lisa Gibbs, Director, Child and Community Wellbeing Unit, Centre for Health Equity, University of Melbourne; and Academic Lead, Community Resilience \& Public Health, Centre for Disaster Management and Public Safety, University of Melbourne, Australia; Richard A. Bryant (D), Scientia Professor and NHMRC Senior Principal Research Fellow, School of Psychology, University of New South Wales, Australia; Cathy Humphreys, Professor of Social Work, Department of Social Work, University of Melbourne, Australia; Kelsey Hegarty, Professor, Centre for Family Violence Prevention, Department of General Practice, The University of Melbourne and The Royal Women's Hospital, Australia;

Connie Kellett, Family Violence Principal Practitioner, Department of Social Work, University of Melbourne; and Victorian Department of Justice and Community Safety, Australia; H. Colin Gallagher, Postdoctoral Researcher, Centre for Transformative Innovation, Faculty of Business and Law, Swinburne University of Technology, Australia; Karen Block, Associate Director, Child and Community Wellbeing Unit, Centre for Health Equity, University of Melbourne, Australia; Louise Harms, Chair and Head, Department of Social Work, University of Melbourne, Australia; John F. Richardson, National Resilience Adviser, Emergency Services, Australian Red Cross, Melbourne, Australia; Nathan Alkemade, Phoenix Australia: Centre for Posttraumatic Mental Health, Department of Psychiatry, University of Melbourne; and Senior Clinical Psychologist, Monash Health, Australia; David Forbes, Director, Phoenix Australia: Centre for Posttraumatic Mental Health, Department of Psychiatry, University of Melbourne, Australia

Correspondence: Robyn Molyneaux. Email: robyn.molyneaux@unimelb.edu.au

First received 11 Jun 2018, final revision 26 Sep 2019, accepted 13 Oct 2019

\section{Funding}

Partner organisations: Outer East Health and Community Support Alliance, Bendigo Loddon Primary Care Partnership, Lower Hume Primary Care Partnership, Central West Gippsland Primary Care Partnership, Lower Hume Primary Care Partnership, Central West Gippsland
Primary Care Partnership, Banyule Nillumbik Primary Care Alliance, Central Hume Primary Care Partnership, Australian Red Cross, Australian Rotary Health, Victorian Department of Health and Centrelink. Beyond Bushfires: Community Resilience and Recovery. LP100200164 \$1 285047 (2010 \$151 116, 2011 \$220 090, 2012 \$232 228.5, 2013 \$301 368, 2014 \$259 179, $2015 \$ 121$ 065.50) Australian Research Council 2009.

\section{Acknowledgements}

The authors gratefully acknowledge the contributions of research participants and the support from community organisations and local governments. The authors also acknowledge the Beyond Bushfires investigators who are not co-authors on this paper but have been important contributors to the overall study: Greg Ireton, Colin MacDougall, Dean Lusher, Pip Pattison and the late Professor Elizabeth Waters. We also acknowledge helpful feedback for this paper from colleague Elena Swift. 


\section{Author contributions}

R.M. contributed to conception and design of paper, conducted analysis and interpretation of data, initial and subsequent drafts of paper, along with revision of article and final approval of the version to be submitted for publication. L.G. contributed to discussions about the conception and design of the paper and the variables for inclusion in the analysis, reviewed and critiqued the draft paper providing constructive suggestions about terminology and interpretation of findings, and provided final approval of the version to be submitted for publication. R.A.B. contributed to conception and design of paper, extensive critical revision of drafts and final approval of version to be submitted for publication. C.H. contributed to discussion of data and paper conception, interpretation of results, critical revision and addition of sion of data and paper conception, interpretation of results, critical revision and addition of
content to article, and final approval of version to be submitted for publication. K.H. contributed to discussion of results and aided in interpretation, critical revision of article and final approval of version to be submitted. C.K. provided subject matter expertise along with interpretation of data and understanding of the significance of the findings, contributed extensive commentary and editing to drafts and approval of final version for submission. H.C.G. guided paper design and the variables to be included in analysis, along with review and interpretation of data analysis, responses to prior reviewer requests and comments, revision of drafts and final approval lysis, responses to prior reviewer requests and comments, revision of drafts and final approval
of the version to be submitted. K.B. contributed to conception and design of the study as well as ongoing management of data collection, critical revision of the article and approval of the submitted version. L.H. contributed to discussions about the conception and design of the paper, reviewed and critiqued the draft paper providing constructive suggestions about the framing and interpretation of findings, and provided final approval of the version to be submitted for publication. J.F.R. contributed to conception and design of the paper, reviewed and critiqued was involved in design and analysis and interpretation of the data, revision of the article and approval of the final version to be submitted for publication. D.F. made substantial contribution to conception and design of the paper, analysis plan, interpretation of data, drafting and revising the article, and final approval of the version to be published.

\section{References}

1 Norris FH, Friedman MJ, Watson PJ, Byrne CM, Diaz E, Kaniasty K. 60,000 disaster victims speak: part I. An empirical review of the empirical literature, 1981-2001. Psychiatry 2002; 65(3): 207-39.

2 Coker AL, Hanks JS, Eggleston KS, Risser J, Tee PG, Chronister KJ, et al. Social and mental health needs assessment of Katrina evacuees. Disaster Manag Response 2006; 4(3): 88-94.

3 Bryant RA, Waters E, Gibbs L, Gallagher HC, Pattison P, Lusher D, et al. Psychological outcomes following the Victorian Black Saturday bushfires. Aust NZ J Psychiatry 2014; 48(7): 634-43

4 Forbes D, Alkemade N, Waters E, Gibbs L, Gallagher C, Pattison P, et al. The role of anger and ongoing stressors in mental health following a natural disaster. Aust NZ J Psychiatry 2015; 49(8): 706-13.

5 World Health Organization (WHO). World Report on Violence and Health: Summary. WHO, 2002 (https://www.who.int/violence_injury_prevention/violence/world_report/en/summary_en.pdf).

6 Heise L, Ellsberg M, Gottmoeller M. A global overview of gender-based violence. Int J Gynecol Obstet 2002; 78: S5-14.

7 Kolbe AR, Hutson RA, Shannon H, Trzcinski E, Miles B, Levitz N, et al. Mortality, crime and access to basic needs before and after the Haiti earthquake: a random survey of Port-au-Prince households. Med Confl Surviv 2010; 26(4): 281-97.

8 Schumacher JA, Coffey SF, Norris FH, Tracy M, Clements K, Galea S. Intimate partner violence and Hurricane Katrina: predictors and associated mental health outcomes. Violence vict 2010; 25(5): 588-603.

9 Sety M, University of New South Wales. Domestic Violence and Natural Disasters. Australian Domestic and Family Violence Clearinghouse, 2012.

10 Frasier PY, Belton L, Hooten E, Campbell MK, DeVellis B, Benedict S, et al. Disaster down East: using participatory action research to explore intimate partner violence in eastern North Carolina. Health Edu Behav 2004; 31(4 Suppl): 69S-84S.

11 Buttell FP, Carney MM. Examining the impact of Hurricane Katrina on police responses to domestic violence. Traumatology 2009; 15(2): 6-9.

12 Anastario $M$, Shehab N, Lawry L. Increased gender-based violence among women internally displaced in Mississippi 2 years post-Hurricane Katrina. Disaster Med Public Health Prep 2009; 3(1): 18-26.

13 Fagen JL, Sorensen W, Anderson PB. Why not the University of New Orleans? Social disorganization and sexual violence among internally displaced women of Hurricane Katrina. J Community Health 2011; 36(5): 721-7.
14 Harville EW, Taylor CA, Tesfai H, Xiong X, Buekens P. Experience of Hurricane Katrina and reported intimate partner violence. J Interpers Violence 2011; 26 (4): 833-45.

15 Lauve-Moon K, Ferreira RJ. An exploratory investigation: post-disaster predictors of intimate partner violence. Clin Soc Work J 2017; 45(2): 124-35.

16 Biswas A, Rahman A, Mashreky S, Rahman F, Dalal K. Unintentional injuries and parental violence against children during flood: a study in rural Bangladesh. Rural Remote Health 2010; 10(1): 1199.

17 Anastario MP, Larrance R, Lawry L. Using mental health indicators to identify postdisaster gender-based violence among women displaced by Hurricane Katrina. J Women's Health 2008; 17(9): 1437-44.

18 Victorian Bushfires Royal Commission. The 2009 Victorian Bushfires Royal Commission Final Report Summary. Victorian Bushfires Royal Commission, 2009 (http://royalcommission.vic.gov.au/Commission-Reports/Final-Report/ Summary.html).

19 Shimizu S, Aso K, Noda T, Ryukei S, Kochi Y, Yamamoto N. Natural disasters and alcohol consumption in a cultural context: the Great Hanshin Earthquake in Japan. Addiction 2000; 95(4): 529-36.

20 Nordløkken A, Pape H, Heir T. Alcohol consumption in the aftermath of a natural disaster: a longitudinal study. Public Health 2016; 132(Supplement C): 33-9.

21 Gilchrist G, Hegarty K, Chondros P, Herrman H, Gunn J. The association between intimate partner violence, alcohol and depression in family practice. BMC Fam Pract 2010; 11(1): 72.

22 Golding JM. Intimate partner violence as a risk factor for mental disorders: a meta-analysis. J Fam Violence 1999; 14(2): 99-132.

23 Gibbs L, Waters E, Bryant RA, Pattison P, Lusher D, Harms L, et al. Beyond Bushfires: Community, Resilience and Recovery-A longitudinal mixed method study of the medium to long term impacts of bushfires on mental health and social connectedness. BMC Public Health 2013; 13: 1036-46.

24 Bliese PD, Wright KM, Adler AB, Cabrera O, Castro CA, Hoge CW. Validating the primary care posttraumatic stress disorder screen and the posttraumatic stress disorder checklist with soldiers returning from combat. J Consult Clin Psychol 2008; 76(2): 272-81.

25 Kroenke K, Spitzer RL, Williams JB. The PHQ-9. J Gen Intern Med 2001; 16(9): 606-13.

26 Bush K, Kivlahan D, McDonell M, Finn S, Bradley K. The AUDIT Alcohol Consumption Questions (AUDIT-C): an effective brief screening test for problem drinking. Arch Intern Med 1998; 158: 1789-95.

27 Gibbs L, Molyneaux R, Whiteley S, Block K, Harms L, Bryant RA, et al. Distress and satisfaction with research participation: impact on retention in longitudinal disaster research. Int J Disaster Risk Reduction 2017; 27: 68-74.

28 Australian Bureau of Statistics. 2011 Census Community Profiles. Australian Bureau of Statistics, 28 March 2013 (https://quickstats.censusdata.abs.gov. au/census_services/getproduct/census/2011/communityprofile/0).

29 Muthén LK, Muthén BO. Mplus User's Guide (6 edn). Muthén \& Muthén, 1998-2011.

30 McNeish D, Stapleton LM, Silverman RD. On the unnecessary ubiquity of hierarchical linear modeling. Psychol Methods 2017; 22(1): 114-140.

31 Fredman SJ, Monson CM, Schumm JA, Adair KC, Taft CT, Resick PA. Associations among disaster exposure, intimate relationship adjustment, and PTSD symptoms: can disaster exposure enhance a relationship? J Trauma Stress 2010; 23(4): 446-51.

32 Taft $\mathrm{CT}$, Monson CM, Schumm JA, Watkins LE, Panuzio J, Resick PA Posttraumatic stress disorder symptoms, relationship adjustment, and relationship aggression in a sample of female flood victims. J Fam Violence 2009; 24(6): 389-96.

33 Galea S, Brewin CR, Gruber M, Jones RT, King DW, King LA, et al. Exposure to hurricane-related stressors and mental illness after Hurricane Katrina. Arch Gen Psychiatry 2007; 64(12): 1427-34.

34 Tharp AT, Vasterling JJ, Sullivan G, Han X, Davis T, Deitch EA, et al. Effects of preand post-Katrina nonviolent and violent experiences on male veterans' psychological functioning. Disaster Med Public Health Prep 2011; 5(Suppl 2): S227-34.

35 Bell SA, Folkerth LA. Women's mental health and intimate partner violence following natural disaster: a scoping review. Prehosp Disaster Med 2016; 31 (6): 648-57. 